\title{
Soluble Angiopoietin Receptor Tie-2 in Patients with Acute Myocardial Infarction and its Detection by Optical Protein-chip
}

\author{
Chun-Ling Wang and Jun-Qiu Li \\ Heart Centre, China Mei-tan General Hospital, Beijing, China
}

\author{
Hong-Wei Li \\ Institute of Microcirculation, Peking Union Medical College \& Chinese Academy of Medical Sciences, Beijing, China
}

Gang Jin, Zhan-Hui Wang and Yong-Hong Meng

National Microgravity Laboratory, Institute of Mechanics, Chinese Academy of Sciences, Beijing, China

\author{
Rui-Juan Xiu \\ Institute of Microcirculation, Penking Union Medical College \& Chinese Academy of Medical Sciences, Beijing, China
}

\begin{abstract}
The Tie-2 receptor has been shown to play a role in angiogenesis in atherosclerosis. The conventional method assaying the level of soluble Tie-2 (sTie-2) was ELISA. However, this method has some disadvantages. The aims of this research are to establish a more simple detection method, the optical protein-chip based on imaging ellipsomtry (OPC-IE) applying to Tie-2 assay. The sTie-2 biosensor surface on silicon wafer was prepared first, and then serum levels of sTie-2 in 38 patients with AMI were measured on admission (day 1), day 2, day 3 and day 7 after onset of chest pain and 41 healthy controls by ELISA and OPC-IE in parallel. Median level of sTie-2 increased significantly in the AMI patients when compared with the controls. Statistics showed there was a significant correlation in sTie-2 results between the two methods $(r=0.923, P<0.01)$. The result of this study showed that the level of sTie-2 increased in AMI, and OPC-IE assay was a fast, reliable, and convenient technique to measure sTie- 2 in serum.
\end{abstract}

Keywords: Soluble Tie-2, acute myocardial infarction, ELISA, optical protein-chip, imaging ellipsometry

\section{INTRODUCTION}

The recently discovered angiopoietins, another family of vascular growth factors, have been identified as playing a crucial role in the completion of angiogenesis. All members of this family are known to bind to the tyrosine kinase receptors Tie-1 or Tie-2, which are expressed only on ECs or early hematopoietic cells $[1,2]$.

Soluble Tie-2 has recently been identified in blood samples from healthy subjects by the enzyme linked immunosorbent assay (ELISA) system $[3,4]$. In the previous study, the variation of sTie-2 was measured in the serum of patients with acute myocardial infarction (AMI) using ELISA [4]. However, this method has some disadvantages; it is time-consuming, has a complicated assaying procedure, needs labeling of samples, and has no unified standard for the results. In the present study, we developed an optical protein-chip based on imaging ellipsometry (OPC-IE) for detecting of sTie- 2 in serum and assessed the feasibility of this label-free method in applying to clinical diagnosis.

\section{MATERIALS AND METHODS}

\section{Materials}

Monoclonal anti-human Tie-2 monoclone antibody, recombinant human Tie-2/Fc chimera, and human Tie-2 
immunoassay quantikine kit were obtained from R\&D System (R\&D Systems, Inc., Minneapolis, MN, USA). Silicon wafers were purchased from General Research Institute for Nonferrous Metals of China. Optical proteinchip biosensor system was provided by Institute of Mechanics, Chinese Academy of Sciences.

\section{Subjects}

Blood samples collected from 41 healthy volunteers with matched criteria of age without cardiovascular disease were used as controls, and 38 patients with AMI admitted to the Coronary Care Unit at Peking Union Medical College Hospital (PUMCH) and Mei-tan General Hospital. Diagnosis of AMI was made according to established criteria of the World Health Organization. Blood samples were collected at the time of admission (1d), 48h (2d), 72h (3d), and 7d after the onset of AMI. Written informed consent was obtained from each subject. We used a serum separator tube (SST) and allowed samples to clot for 30 minutes before centrifugation for 15 minutes at $3000 \mathrm{rpm}$. Serum samples were then aliquoted and stored at $-80^{\circ} \mathrm{C}$ until the time of analysis. Serum samples were diluted 1:10 or 1:2 for the analysis.

\section{ELISA Assay}

sTie-2 concentration in serum was determined using a specific enzyme linked immunosorbent assay (ELISA) kit. Measurements were performed according to the manufacturer's instructions. Standards and samples were incubated on a microplate for 2 hours at room temperature to allow adsorption of sTie- 2 molecules to the immobilized primary antibody. The plate was washed after incubation and blocking period. A mouse monoclonal antibody against Tie-2 conjugated to horseradish peroxidase was added to all wells and incubated for 2 hours at room temperature, then aspirated and washed 4 times. A mixture of two color reagents, A and B, was added to each well and we gently tapped the plate to ensure thorough mixing, then incubated it for 25 minutes at RT. The color development was stopped and photometric quantification was performed by determining the optical density using a microplate reader set to $450 \mathrm{~nm}$. We averaged the duplicated readings for each standard and sample and subtracted the average zero standard optical density. The standard curve was linear from 0 to $100 \mathrm{ng} / \mathrm{mL}$ of Tie- 2 .

\section{OPC-IE Assay}

This system consists of an optical protein-chip, a microinteractor with micro-fluidics system, and an optical reader with imaging ellipsometry [5,6]. The steps of OPC-IE assaying included several sections, as follow:

1. Preparation of optical substrates. It has been mentioned in detail elsewhere [5.6]. Hydrophobic silicon wafers were used for measurement.

2. Preparation of protein-chip with anti-sTie-2 biosensor surface by pre-disposition with anti-sTie-2 monoclone antibody. After definition of homogeneous and cleaning of the wafer surface, the anti-Tie-2 antibody $(0.1 \mathrm{mg} / \mathrm{ml})$ was immobilized to the silicon wafer's surface by a micro-fluidics system, then the hydrophobic surface was incubated with anti-Tie-2 solution for $20 \mathrm{~min}$ at RT, and rinsed with $\mathrm{ddH}_{2} \mathrm{O}_{2}$ for $5 \mathrm{~min}$.

3. Assay of sTie-2 in serum. After blocking for $30 \mathrm{~min}$ at RT with 3\%BSA in PBS-T $(0.05 \%$ Tween-20 in $0.01 \mathrm{M}$ PBS, $\mathrm{pH} 7.2,20 \mu \mathrm{l} / \mathrm{well})$, the Tie-2/Fc protein (ranging from $0 \mathrm{ng} / \mathrm{ml}$ to $50 \mathrm{ng} / \mathrm{ml}$ ) or $1: 1$ diluted serum samples were added in triplicate $(50 \mu \mathrm{l} / \mathrm{well})$ for $40 \mathrm{~min}$ at RT. $100 \mu \mathrm{lddH}_{2} \mathrm{O}_{2}$ was then added for a further rinse to remove all the non-absorbed molecules. We took down the chips and dried with nitrogen.

This complex layer was visualized by the Optical Reader. In this system, the thickness of antigenantibody complex can be observed directly. The surface thickness (or surface density) of analytical areas was measured and expressed in gray scale. The higher the concentration of sTie-2 in serum, the thicker the antigen-antibody complex thickness, and the higher the gray scale.

4. Evaluation of precision and sensitivity. Three samples of known concentration were tested eight times on one chip to assay the inter-assay precision. Three samples of known concentration were tested in 15 separate chips to assay the intra-assay precision. The minimum detectable dose (MDD) was determined by adding two standard deviations to the mean gray scale value of 20 zero standard replicates and calculating the corresponding concentration.

5. Standard curve of sTie-2. We averaged the three triplicate gray scales for each standard and subtracted the average zero standard gray scale. We plotted the gray scale for the standards versus the concentration of the standards and drew the best curve.

6. Calculation of results: we read the corresponding sTie-2 concentration on the standard curve.

\section{Statistical Analysis}

Statistical analyses were performed using the statistical program SPSS 11.0 for Windows (SPSS Inc., Chicago, IL, USA). In the clinical study, data for sTie-2 were nonparametrically distributed and therefore Tie-2 is expressed as median (interquartile range, IQR). Comparison between 
two methods was made using bivariate Spearman's correlation test. A $P$ value of $<0.05$ was considered as statistically significant.

\section{RESULTS}

\section{Clinical Characteristics of Patients and ELISA Assay}

The characteristics for all subjects in the study and results of sTie- 2 have been summarized elsewhere [4].

\section{The Related Data of ELISA Assaying sTie-2 in Serum}

The inter-assay variation and intra-assay variation afforded by the manufacturer are summarized in Table 1 . Three samples of known concentration were tested 20 times on one plate to assay the inter-assay precision. Three samples of known concentration were tested in 40 separate plates to assay the intra-assay precision. The ratio of SD and mean was coefficient of variability $(\mathrm{CV})$, representing the relative variability around the mean. The MDD was $0.014 \mathrm{ng} / \mathrm{ml}$.

\section{Related Data of OPC-IE Assaying sTie-2 in Serum}

The inter-assay and intra-assay precision are summarized in Table 1 . MDD was $2.5 \mathrm{ng} / \mathrm{ml}$.

Nine analytical areas of a protein-chip are shown in Figure 1. When the protein chip is incubated with serum that contains sTie-2, sTie-2 will bond with anti-Tie-2 immobilized on the protein chip to form complexes that cause the thickness increase on the sensing surface. The visualization of the protein layer thickness can be performed and quantified using the optical reader.
A significant increase of the layer thickness indicates that the solution contains the corresponding specific antigen. The gray scale is proportional to the thin-layer thickness. A three-dimensional format image of the result is shown in Figure 1B. The average intensities in gray scale of protein layers of every group are 1d 89.26 (78.78-100.33), 2d 91.17 (86.03-103.5), 3d 89.05 (83.5-99.98), 7d 84.57 (72.55-90.15). Control group is 78.45 (74.3-79.6) (results expressed as median (interquartile range)).

After converting the gray scale into mass concentration, the bivariate Spearman's correlation test showed there was significant correlation in sTie-2 results between the two methods $(r=0.923, P<0.01)$.

\section{DISCUSSION}

The significance about the elevated sTie-2 in AMI has been discussed elsewhere [4]. In this study, we first use the OPC-IE to assay the serum levels of sTie-2 in AMI patients. This system mainly consists of an optical protein-chip and imaging ellipsometry. Imaging ellipsometry is a recently developed optical technique that was used to assay the ultrathin layer. This technique platform was provided by the Institute of Mechanics of Chinese Academy of Sciences. The system used a polarized beam as a detection beam to irradiate samples. The reflection beam carried information of samples passed through an analyzer and an imaging lens, and then the ellipsometric image was focused onto the sensing area of the charge-coupled device (CCD). Combined with image processing techniques, the video signal corresponding to the thickness of the complex layer was captured, digitized, and stored in gray scale format on a computer $[5,6]$. The gray scale is positive proportional to the thinlayer thickness (or biomolecule surface concentration).

Table 1. The inter-assay and intra-assay variation of ELISA and OPC-IE for detection of sTie-2

\begin{tabular}{|c|c|c|c|c|c|c|}
\hline \multirow[b]{2}{*}{ ELISA } & \multicolumn{4}{|c|}{ Inter-assay variation } & \multicolumn{2}{|c|}{ Intra-assay variation } \\
\hline & & & & & & \\
\hline Sample & 1 & 2 & 3 & 1 & 2 & 3 \\
\hline $\mathrm{N}$ & 20 & 20 & 20 & 40 & 40 & 40 \\
\hline Mean concentration $(\mathrm{ng} / \mathrm{ml})$ & 0.76 & 2.57 & 5.14 & 0.82 & 2.65 & 5.15 \\
\hline Standard deviation & 0.04 & 0.11 & 0.26 & 0.07 & 0.17 & 0.27 \\
\hline $\mathrm{CV}(\%)$ & 4.6 & 4.4 & 5.0 & 8.3 & 6.5 & 5.2 \\
\hline \multicolumn{7}{|l|}{ OPC-IE } \\
\hline Sample & 1 & 2 & 3 & 1 & 2 & 3 \\
\hline $\mathrm{N}$ & 8 & 8 & 8 & 15 & 15 & 15 \\
\hline Mean concentration $(\mathrm{ng} / \mathrm{ml})$ & 4.25 & 16.82 & 28.91 & 3.89 & 14.81 & 26.33 \\
\hline Standard deviation & 0.46 & 2.05 & 3.85 & 0.34 & 1.45 & 1.84 \\
\hline $\mathrm{CV}(\%)$ & 10.8 & 12.2 & 13.3 & 8.7 & 9.8 & 7.0 \\
\hline
\end{tabular}


A

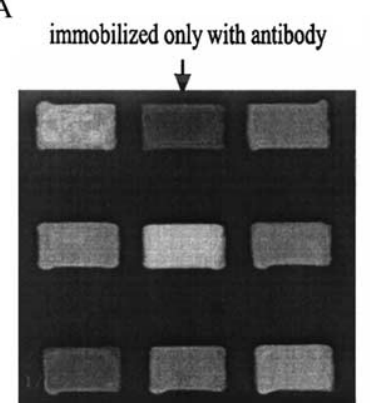

B

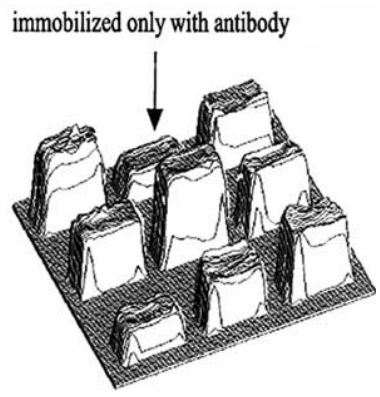

Figure 1. Optical protein-chip based on imaging ellipsometry for detection of sTie-2. (A) Image of the protein chip in gray scale format. (B) Three-dimensional image of the protein chip. Arrow area immobilized with antibody was used as reference. Other areas were added with different serum samples. After incubation with serum solution containing Tie-2, the thickness of the thin-layer increased (B).

When compared with ELISA, the OPC-IE described here has several substantial advantages over ELISA: 1) Results of the OPC-IE assay have a good correlation with ELISA. 2) Compared to other solid-phase methods currently used, such as ELISA, immunofluorescence, and radioimmunoassay, this method has the advantage of not involving any labeling of the reactant. Kodadek [7] pointed out that chemical labeling of proteins can greatly change their surface characteristics, which can result in significant protein denaturation, especially for smaller proteins and peptide hormones, and the process of labeling is a labor-intensive step for a high-throughput protein chip. Therefore, this method avoided disturbances from conjugated markers or handling with radioactive materials and largely simplified the detection operation, so that the detection time can be largely reduced. 3) Imaging ellipsometry is an enhancement of standard single-beam ellipsometry, which uses a charge coupled device (CCD) camera to image the ellipsometric response of a large area on the sample surface. It can monitor multiple proteins or multiple samples simultaneously in real time. These advantages make the protein-chip potentially suited as a promising high-throughput screening technique. 4) Real-time monitoring for protein interactions. The function of real-time analysis for antigen-antibody interaction supplies some important kinetic data, such as the interaction rate and conditions. As one kind of protein chip, the OPC-IE has been used in some biological fields [8]. With the perfection of this technique, we believe it will be used in many fields, such as clinical diagnosis, molecular biology, biochemistry, cell biology, and pharmacology, as a complement to ELISA or immunofluorescence.

Further studies will have to enhance the precision and sensitivity of OPC-IE by modifying the surface of the

silicon wafer. One mixed silanes layer contained two kinds of compounds, 3-aminopropyltriethoxysilane (APTES) and methyltriethoxysilane (MTES) or glutaraldehyde (Glu), was used to modify silicon surface for protein immobilization, and the results showed that the surface modification method could be used to optimize the sensitivity of OPC-IE $[9,10]$.

This work was supported by the "Knowledge Innovative Project of Chinese Academy of Sciences (No. KJCX1SW-07).".

Declaration of interest: The authors report no conflicts of interest. The authors alone are responsible for the content and writing of the paper.

\section{REFERENCES}

1. Dumont, D.J., Gradwohl, G.J., Fong, G.H., Auerbach, R., Breitman., M.L. (1993). The endothelial-specific receptor tyrosine kinase, tek, is a member of a new subfamily of receptors. Oncogene. 8:1293-301.

2. Iwama, A., Hamaguchi, I., Hashiyama, M., Murayama, Y., Yasunaga, K., Suda, T. (1993). Molecular cloning and characterization of mouse TIE and TEK receptor tyrosine kinase genes and their expression in hematopoietic stem cells. Biochem. Biophys. Res. Commun. 195:301-9.

3. Chung, N.A.Y., Makin, A.J., Lip, G.Y.H. (2003). Measurement of the soluble angiopoietin receptor tie-2 in patients with coronary artery disease: development and application of an immunoassay. Eur. J. Clin. Invest. 33:529-535.

4. Wang, C., Fu, P., Li, H., Gao, R., Xiu, R. (2005). Soluble Tie-2 in patients with acute myocardial infarction and its effects on angiogenesis. Clin. Hemorheol. Micro. 33:1-10.

5. Wang, Z., Jin, G. (2003). Feasibility of protein A for the oriented immobilization of immunoglobulin on silicon surface for a biosensor with imaging ellipsometry. J. Biochem. Biophys. Methods. 57:203-11.

6. Wang, Z.H., Jin, G. (2003). A label-free multisensing immunosensor based on imaging ellipsometry. Anal. Chem. 75:6119-6123.

7. Kodadek, T. (2001). Protein microarrays: prospects and problems. Chem. Bio. 8:105-15.

8. Tao, S.C., Chen, C.S., Zhu, H. (2007). Applications of protein microarray technology. Comb Chem High Throughput Screen. 10:706-718.

9. Zhang, H.G., Qi, C., Wang, Z.H., Jin, G., Xiu, R.J. (2005). Evaluation of a new CA15-3 protein assay method: optical protein-chip system for clinical application (Part I). Clin. Chem. 51:1038-1040.

10. Wang, Z.H., Meng, Y.H., Ying, P.Q., Qi, C., Jin, G. (2006). A label-free protein microfluidic array for parallel immunoassays. Electrophoresis. 27:4078-4085. 
Copyright of Artificial Cells, Blood Substitutes, \& Biotechnology is the property of Taylor \& Francis Ltd and its content may not be copied or emailed to multiple sites or posted to a listserv without the copyright holder's express written permission. However, users may print, download, or email articles for individual use. 